\title{
The impact of the heart failure health enhancement program: A retrospective pilot study
}

\author{
Cynthia J. Hadenfeldt, Marilee Aufdenkamp, Caprice A. Lueth, Jane M. Parks \\ Creighton University College of Nursing - Hastings Campus, Hastings, Nebraska
}

Correspondence: Cynthia J. Hadenfeldt. Address: Creighton University College of Nursing - Hastings Campus, Hastings, Nebraska. Email: cynthiahadenfeldt@creighton.edu

Received: November 27, 2013

Accepted: February 6, 2014

Online Published: February 17, 2014

DOI : $10.5430 /$ cns.v2n2p51

URL: http://dx.doi.org/10.5430/cns.v2n2p51

\begin{abstract}
Background: Heart Failure (HF) is a health care issue associated with high rates of morbidity and mortality, costing the United States billions of dollars annually. Current healthcare reform requires managing transitions from acute care to home so that the disease is managed effectively and readmissions prevented. Routine HF discharge care includes education regarding prescribed medications, weight monitoring, dietary restrictions, and symptom exacerbation. The Heart Failure Health Enhancement Program (HFHEP) was developed to provide outpatient education and support to assist patients in implementing practices designed to decrease exacerbations of heart failure and prevent readmissions.
\end{abstract}

Purpose: This retrospective pilot study evaluated the effectiveness of the HFHEP intervention after completion of the program's first year. The specific aims of the study were to examine differences in inpatient readmission rates within 30 days and from 31 to 90 day for individuals diagnosed with HF following hospital discharge during the intervention year and the year prior to the intervention, and to examine differences between participants and non-participants of the HFHEP program during the intervention year. An additional aim was to determine whether individuals' demographic characteristics and left ventricular ejection fraction (LVEF), as a measure of severity of illness, differed between the two periods and influenced participation in the HF program and readmission outcomes. The final aim was to determine if common barriers prevented participation in the program.

Methods: The study, which utilized a retrospective design, was conducted at a 118 bed community hospital in the Midwest. Two hundred eleven patients generated 266 acute care stays with a primary diagnosis of HF during the two years. Intervention year data was compared to data from the year prior to the intervention. Descriptive statistics and chi square analysis were used to analyze the data.

Results: While not achieving statistical significance, there were fewer readmissions within 30 days of discharge during the intervention year (5\%) compared to the non-intervention year $(9.6 \%)$ representing a nearly $50 \%$ decrease in readmissions. No statistically significant findings were demonstrated between individual's LVEF and 30-day readmissions, 31-90 day readmissions, or program participation. Major barriers to participation included nursing home residency, lack of referral, patient refusal, patient expiration, and no transportation to the program. An unexpected finding was that $14 \%$ of the patients had LOS exceeding the expected LOS for HF.

Conclusions: The HFHEP was an effective intervention to prevent 30-day readmission for patients discharged with a primary diagnosis of HF; however, the number of participants was insufficient to achieve statistical significance. Recommendations of the study include adding a tele-monitoring component to the intervention, automating the referral 
process to capture all potential participants, providing educational programs for nursing home and assisted living personnel regarding best practices in the management of $\mathrm{HF}$, and further investigation of reasons for patient refusal to participate in the HFHEP. Recommendations for future research are also discussed.

\section{Key words}

Heart failure, Nursing, Readmission, Education, Nurse-managed

\section{Introduction}

Heart failure (HF), a disease process with multiple underlying causes, is an important health care issue associated with high rates of morbidity and mortality ${ }^{[1-3]}$. It affects approximately five million people in the United States ${ }^{[4]}$ and is, by its nature, a chronic debilitating disease. Heart failure occurs when the heart is unable to function properly due to myocardial damage and is most commonly treated with behavioral interventions and with medications to minimize fluid volume and strengthen myocardial contractility. Mortality related to heart failure is significant as $80 \%$ of men and $70 \%$ of women over 65 years of age die within 8 years of diagnosis ${ }^{[3]}$. Despite efforts on the part of health care organizations to prevent readmissions by implementing comprehensive discharge teaching and multidisciplinary HF management programs ${ }^{[2,5]}$, unplanned hospital readmissions cost Medicare $\$ 17.4$ billion a year with $\$ 12$ billion spent annually on potentially preventable admissions ${ }^{[6]}$. Nationally, $26.9 \%$ of Medicare enrolled HF patients are readmitted within 30 days of a hospital discharge for $\mathrm{HF}^{[6]}$. It is important to note that fifty-four percent of these readmissions are preventable when patients regularly monitor their health and make behavior changes to prevent heart failure exacerbations ${ }^{[5]}$.

An essential component of care for the patient with heart failure is providing education that promotes compliance with medication regimens, daily weight monitoring, dietary restrictions, and assists the individual in rapidly identifying symptoms that may indicate an exacerbation of their illness potentially preventing a readmission to the hospital. Despite discharge education being provided at the conclusion of most hospital stays, additional education and support, in the weeks and months following discharge, are often needed to maintain health and prevent readmission.

Research demonstrates that outpatient nurse-led HF disease management programs can decrease costs related to HF and prevent readmissions. These outpatient programs aid patients in understanding and implementing strategies to improve quality of life, prevent readmissions, and can be individualized to meet the patient's needs ${ }^{[1,3-5]}$. Outpatient HF disease management programs can be a cost effective measure to improve patient outcomes and increase patient satisfaction with care. In addition to providing benefit to patients, current proposals in healthcare reform and proposed payment models create a compelling case to manage transitions from acute care so that the disease can be managed more effectively and costs can be controlled.

The Heart Failure Health Enhancement Program (HFHEP) was developed to provide additional outpatient education to patients discharged with a primary diagnosis of HF from a 118-bed community hospital located in the Midwestern United States. The HFHEP had not been formally evaluated prior to this study. In addition to collecting readmission data, demographic characteristics were collected to determine if patients during the non-intervention year were similar to those during the intervention year and if they might influence participation in the program. Left ventricular ejection fraction (LVEF) was collected as a measure of severity of illness to determine if there was a correlation between LVEF and associated readmissions. Patient reported barriers to participation in the program were identified to determine whether there were common barriers that hindered participation that could be alleviated in future years of the intervention.

The purpose of this retrospective pilot study was to determine the effectiveness of the intervention one year after implementation. The specific aims of the study were to examine: 
- Differences in inpatient readmission rates within 30 days and from 31 to 90 days for individuals diagnosed with HF following hospital discharge during the intervention year compared to the non-intervention year.

- Differences in inpatient readmission rates within 30 days and from 31 to 90 days for participants in the HFHEP and those who did not participate in the program.

- Whether demographic characteristics (e.g., age, gender, marital status, and living situation) and LVEF differed between the two periods (e.g., the intervention and non-intervention years) and to examine if demographic characteristics and LVEF of patients who participated in the HFHEP differed from non-participants during the intervention year.

- Common barriers preventing participation in the program.

\section{Theoretical framework}

The theoretical framework for this study was the Health Belief Model, which emphasizes the importance of the individuals' perception of threats to their health and of the individuals' confidence in their ability to change the outcome of the disease by changing their behavior ${ }^{[7]}$. The model describes "modifying variables" that factor into the individual's perception that they are at risk for a serious illness ${ }^{[7]}$. Perceived benefits and barriers are an integral part of the model as well as cues to action that assist the individual in deciding that they should implement changed behaviors as soon as possible to prevent illness exacerbation ${ }^{[7]}$.

The model was chosen for this study because the HFHEP was designed to modify the beliefs of the individual by providing information and support in order to influence changes in unhealthy behaviors. In addition to focusing on readmissions for HF, this study focused on identifying variables such as demographic characteristics, severity of illness, and perceived barriers that might have influenced participation in the intervention and that could be altered to increase future enrollment of HF patients in the program.

\section{Literature review}

Best practices in heart failure disease management include equipping patients with the tools needed to manage their illness upon discharge. As a measure of quality and impacting reimbursement for care, hospitals are required to prevent readmissions following hospital discharge in patients with heart failure. Outcome measures, and strategies to achieve these outcomes, have been reported in the literature ${ }^{[3,8-11]}$. Overall patient mortality, mortality due to heart failure, hospital lengths of stay, costs of hospital care, number of hospital readmissions, quality of life indicators, functional class measurement, emergency department visits, patient satisfaction, and changes in morbidity due to disease progression were the outcomes of interest. In these studies strategies for disease management included physician-led or nurse-led educational programs. However, the majority of interventions represented multidisciplinary collaboration with nurses, physicians, pharmacists, dietary, and social services contributing by assisting patients through education, review of medications and medication adjustments, promotion of dietary restrictions and exercise regimens, and helping patients' access resources. Education was provided in the hospital during discharge, during home visits, in clinic settings, and by telephone through structured weekly calls made by the nurse or by making a 24-hour telephone line available to the patient to call in. Interventions ranged from one home visit to 12 months of follow-up.

Krumholz ${ }^{[9]}$ and Dunagan ${ }^{[10]}$ found that outpatient educational and support interventions by specially trained or experienced cardiac nurses were effective strategies in decreasing heart failure readmissions. Interventions included nurse telephone calls at prescribed intervals, which focused on supporting patient self-management of heart failure. Additionally, nurses in the Dunagan study ${ }^{[10]}$ screened patients for symptoms and adjusted the diuretic dosage according to a protocol or contacted the patients' physicians for diuretic adjustment. Both studies compared readmission rates of the intervention group against a control group. Readmission rates for the intervention group were found to be reduced 
compared to the control groups. The Krumholz study demonstrated significance in lowered HF readmission rates and mortality in the intervention group over the course of a year ${ }^{[9]}$.

Stauffer ${ }^{[12]}$ found that a transitional care program led by Advanced Practice Nurses (APN), which included eight home visits by an APN over a three-month period resulted in a statistically significant reduction in 30-day post-intervention HF readmission rates. The initial home visit occurred within 72 hours of discharge from the hospital. All visits focused on protocol management, goal setting, and education. Follow-up phone calls were available.

\section{Methods}

This retrospective study was approved by the academic Institutional Review Board and consent obtained from the institutional facility to conduct the study. In order to determine the effectiveness of the intervention in reducing readmissions, results were compared from the first year the program was implemented (April 2011-April 2012) to the year immediately prior to intervention (April 2010-April 2011). Data was collected and analyzed with regard to demographics (gender, age, marital status, and living situation), number of hospital readmissions at 30 and 31 to 90 days, length of hospital stay, and LVEF (as a measure of severity of illness). Additionally, during the intervention year, data was collected regarding participation or non-participation in the HFHEP, number of sessions attended, and barriers to participation.

\subsection{Sample}

Two registered nurses from the health care facility completed a retrospective collection of data utilizing an Excel spreadsheet designed by the researchers. The nurses were familiar with the hospital electronic medical record and where to find the required data. They were trained by investigators on the method to code each entry. All patient identifiers were removed prior to review of the data by study investigators. Inclusion criteria included all patients discharged with a primary diagnosis of heart failure from April 2010 to April 2012. Data was collected on 211 patients who generated 266 admissions.

\subsection{I ntervention}

The intervention consisted of six hourly outpatient educational sessions with a specially trained cardiac rehab nurse for the patient and family, if desired. One session of physician education on best practices of medication therapy for HF patients prefaced the initiation of the program. Outpatient sessions were offered to patients discharged with a primary diagnosis of HF from April 2011 to April 2012. The sessions were individualized and scheduled weekly for four weeks, then every other week for four weeks. The program was flexible in providing sessions adapted to the severity of the HF, number and severity of co-morbid conditions, degree of social support, and educational background of the individual patient. The Cardiac Rehabilitation nurses visited each heart failure patient during their hospitalization to introduce the program, determine their interest in participation, and schedule the patient for a brief history intake prior to beginning the HFHEP. The intervention utilized a study book called "A Stronger Pump". Topics included HF pathophysiology, reportable signs and symptoms, diagnostic tests, daily weight monitoring, and medication and behavioral treatment interventions, including sodium and fluid restrictions.

Appointments were arranged at the patient's convenience, generally one-week post hospitalization, to begin the educational sessions in the cardiac rehab area of the hospital. Since daily weight monitoring is essential for management, digital scales were provided to patients who did not have one. During the educational sessions, the nurse assessed the patients for signs of exacerbation, compliance with weight monitoring, and compliance with their medication regimen. A multidisciplinary approach was utilized during the one-on-one sessions with participants meeting with diabetic educators, dieticians, pharmacists, and respiratory therapists for assessment and additional education as needed. 


\subsection{Data analysis}

Data was transferred from the Excel spreadsheet to SPSS version 20.0 software for analysis. Descriptive statistics were calculated including frequency distributions and percentages and chi-square analysis was performed to determine statistical significance between the groups of patients during the intervention year and the year prior to the intervention, and between participants and non-participants in the HFHEP during the intervention year. Chi square was also used to compare patients with LVEF $>40$ to those with LVEF $<40$ and readmission within 30 days, readmissions within 31-90 days, and participation in the program. Statistical significance was assessed at an alpha level of 0.05 .

\section{Results}

\subsection{Demographic characteristics}

Demographic characteristics of age, gender, marital status, and living situation were analyzed utilizing descriptive statistics for the intervention year and the prior year (see Table 1). There were 93 patients discharged with a primary diagnosis of HF from April 2010-April 2011 and 118 patients discharged from April 2011-April 2012. Fourteen of these 211 patients had hospitalizations during both the non-intervention and intervention years. There were no statistically significant differences between the patient demographics of gender and marital status during the non-intervention and intervention years. There was a statistically significant difference $(p=0.01)$ for living situation (independent living vs. living with assistance) with intervention year patients more likely to live independently than live with assistance (nursing home, assisted living, hospice).

Table 1. Demographic characteristics of the sample $(\mathrm{N}=211)$

\begin{tabular}{|c|c|c|c|c|}
\hline & Year prior to intervention & Intervention year & \multirow{3}{*}{ Chi square } & \multirow{3}{*}{$P$ value } \\
\hline & April 2010-April 2011 & April 2011-April 2012 & & \\
\hline & $\mathbf{N}=93$ & $\mathrm{~N}=118$ & & \\
\hline Variable & $\mathrm{n}(\%)$ & $\mathrm{n}(\%)$ & & \\
\hline Gender & & & $\chi^{2}(1, n=.211)=.633$ & .633 \\
\hline Female & $48(52 \%)$ & $57(49)$ & & \\
\hline Male & $45(48 \%)$ & $61(51)$ & & \\
\hline Mean Age & $81 \pm 12$ years & $77 \pm 12$ years & & \\
\hline Marital Status & & & $\chi^{2}(1, \mathrm{n}=211)=763$ & .382 \\
\hline Married & $41(44 \%)$ & $45(38)$ & & \\
\hline Single & $52(56 \%)$ & $73(62)$ & & \\
\hline Living Situation & & & $\chi^{2}(1, \mathrm{n}=200)=5.457$ & .019 \\
\hline Independent Living & $44(47 \%)$ & $76(64)$ & & \\
\hline Living with Assistance & $43(46 \%)$ & $37(32)$ & & \\
\hline Expired & $6(7 \%)$ & $5(4)$ & & \\
\hline Total Admissions & 121 & 145 & & \\
\hline LVEF & & & $\chi^{2}(1, \mathrm{n}=188)=.026$ & .871 \\
\hline $\mathrm{LVEF} \leq 40$ & 54 & 71 & & \\
\hline LVEF $>40$ & 28 & 35 & & \\
\hline Missing LVEF & 11 & 12 & & \\
\hline
\end{tabular}


There were 22 participants in the HFHEP and 96 non-participants during the intervention year. There were no statistically significant differences in patient demographics (age, gender, marital status, and living situation) between participants and non-participants during the intervention year, however, participants were more likely to be male, married, and living independently (see Table 2).

Table 2. Demographic characteristics of program participants and non-participants ( $\mathrm{N}=118)$

\begin{tabular}{|c|c|c|c|c|}
\hline & Participant & Non-participant & & \\
\hline & $\mathrm{N}=\mathbf{2 2}$ & $\mathrm{N}=96$ & & $P$ vatue \\
\hline Variable & $\mathrm{n}(\%)$ & $\mathrm{n}(\%)$ & & \\
\hline Gender & & & $\chi^{2}(1, \mathrm{n}=118)=1.544$ & .214 \\
\hline Female & $8(36 \%)$ & $49(51 \%)$ & & \\
\hline Male & $14(64 \%)$ & $47(51 \%)$ & & \\
\hline Mean Age & 74 years & 78.7 years & & \\
\hline \multicolumn{5}{|l|}{ Marital Status } \\
\hline Married & $12(55 \%)$ & $33(34 \%)$ & $\chi^{2}(1, \mathrm{n}=118)=3.087$ & .079 \\
\hline Single & $10(45 \%)$ & $63(66 \%)$ & & \\
\hline Living Situation & & & $\chi^{2}(1, \mathrm{n}=118)=3.576$ & .059 \\
\hline Independent Living & $19(86 \%)$ & $57(59 \%)$ & & \\
\hline Living with Assistance & $3(14 \%)$ & $34(35 \%)$ & & \\
\hline LVEF & & & $\chi^{2}(1, \mathrm{n}=106)=2.524$ & .112 \\
\hline $\mathrm{LVEF}>40$ & 11 & 60 & & \\
\hline LVEF $<40$ & 10 & 25 & & \\
\hline Missing LVEF & 1 & 11 & & \\
\hline
\end{tabular}

\subsection{Comparison of readmission data}

There were nine patients $(9.6 \%)$ who had at least one readmission in less than 30 days during the non-intervention year and six patients (5\%) who had at least one readmission in less than 30-days during the intervention year representing a nearly $50 \%$ decrease in readmissions from the non-intervention year to the intervention year. Eleven patients $(11.7 \%)$ had at least one readmission between 31-90 days of discharge during the non-intervention year and 12 patients $(10 \%)$ had at least one readmission between 31 and 90 days during the intervention year.

\subsection{Participation in the program}

During the intervention year, there were 22 participants and 96 non-participants. One participant had at least one readmission within 30 days (4.5\%) and five participants had at least one readmission within 31-90 days (22.7\%). Of the non-participants, five had at least one readmission within 30-days (5\%) and seven had at least one readmission with 31-90 days $(7 \%)$. There were no statistically significant differences between participants and non-participants regarding readmissions.

\subsection{Barriers}

Barriers to participation in the HFHEP are identified in Figure 1. The top barriers were nursing home residency, refusal to participate, lack of referral to the program, no transportation to the program, and patient death (expiration). Although not excluded from the program, nursing home residents did not participate possibly due to the provision of 24 hour nursing care and lack of transportation for an outpatient program. Additional barriers included admission to an assisted living 
facility, readmission to the hospital, and failure to present for the scheduled HFHEP sessions with the reason being unknown.

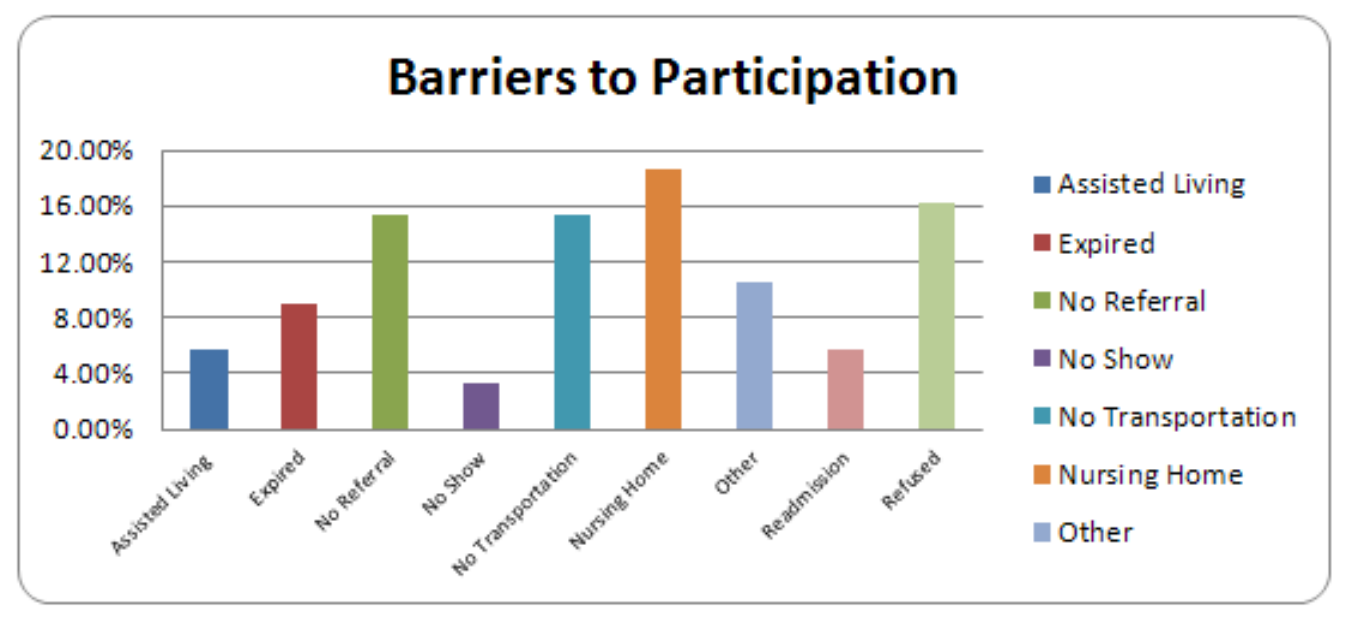

Figure 1. Barriers to participation

\subsection{Left ventricular ejection fraction}

LVEF as a measure of severity of illness was available in the medical records for 189 of the 211 patients and are displayed in the tables as LVEF $>40$ representing normal contractility and LVEF $<40$ which indicates decreased contractility and a compromised myocardium. Patients with an LVEF $>40$ were compared to those patients with LVEF $<40$ with regard to 30 -day readmission $\chi^{2}(1, \mathrm{n}=189)=.154, p=.695,31-90$ day readmission $\chi^{2}(2, \mathrm{n}=189)=2.029, p=.363$, and participation in the program $\chi^{2}(1, \mathrm{n}=118)=.2 .524, p=.112$. No statistical significance was demonstrated in any of these analyses.

\subsection{Length of stay}

An unexpected finding during the two years was that 31 subjects (14\%) had hospital LOS exceeding the expected LOS for HF. Of the 31 subjects who had a longer LOS (7-13 days), $70 \%$ were over 80 years of age, $63 \%$ were single, $87 \%$ had no readmissions in $<30$ days, $77 \%$ had an LVEF of $>40,56 \%$ lived independently, and $30 \%$ resided in nursing homes. Older age and being single increased the likelihood of having a longer LOS. Patients with a longer LOS were less likely to readmit within 30 days.

\section{Discussion}

The primary aim of this retrospective pilot study was to determine whether there was a difference in inpatient readmission rates within 30 days and from 31 to 90 days for patients discharged with a primary diagnosis of HF during the intervention year compared to the non-intervention year. While the intervention year had an increased number of admissions overall (145 compared to 121 in the non-intervention year), the rate of readmission within 30-days was decreased during the intervention year compared to the non-intervention year. There were no significant decreases in readmissions during the 31-90 day period between the two years.

The second aim was to determine whether patients who participated in the HFHEP had improved readmission outcomes compared to patients who did not participate in the program during the intervention year. During the intervention year, there were 22 participants and 96 non-participants to the program. The intervention was effective as evidenced by the readmission of only one of the 22 participants; however, the number of participants was low and statistical significance was not achieved. With respect to participant vs. non-participants during the intervention year, there were no significant differences in the readmission rates at 30 -days $\chi^{2}(1, \mathrm{n}=118)=.016, p=.898$. There was an unexpected inverse finding for 
readmissions at 31-90 days between these two groups (22\% for participants and $7 \%$ for non-participants). Additional study with a larger sample size is recommended to further evaluate the effectiveness of the program in decreasing readmission rates.

The third aim was to determine whether demographic characteristics (age, gender, marital status, and living situation) and LVEF were comparable between the two years and to determine whether demographic characteristics and LVEF of patients who participated in the HFHEF differed from non-participants during the intervention year. Demographic characteristics were similar during both the intervention and non-intervention year with the exception that patients during the intervention year were more independent in their living situation than during the non-intervention year. Even though patients discharged to nursing homes were not excluded from the HFHEP, none participated. This is consistent with other studies ${ }^{[9,10]}$ where nursing home patients were excluded from heart failure outpatient educational programs because health care providers were responsible for their daily care and the patients could not comprehend or had no need to receive further instruction. Not all patient medical records reported a measure of LVEF, for the 189 that did, there was no correlation between LVEF and readmission at 30-days, at 31-90 days, participation in the program or LOS. Continued evaluation of demographic characteristics is recommended to determine homogeneity of sample and facilitate future statistical analysis.

The fourth aim was to determine if there were common barriers to participation in the program. The top barriers were nursing home residency, lack of referral, refusal to participate, no transportation, and patient death (expiration). Nursing home residency was a barrier to participation in the program as patients who reside in nursing homes generally cannot manage their own care and rely on others to care for them. Heart Failure patients experiencing cognitive impairment were also not referred to the program because they were unable to participate due to their inability to follow through with instruction. Lack of referral was a common barrier to participation in the HFHEP though referral was triggered by use of standardized heart failure orders and the program was of no cost to the patient. It was not discovered until collection of data that there was a problem with the referral process and a number of potential patients were not invited to participate in the program. Barriers were classified as "no transportation" if potential participants lived at distance from the hospital where the education was being held or if they were located out of state and had been hospitalized while they were visiting in the area. Some patients expired following discharge without an opportunity to participate.

In spite of research supporting the benefits of HF disease management programs, enrollment and participation in outpatient educational programs remains consistently low ${ }^{[13]}$. In addition to the lack of participation on the part of the patients themselves, one study ${ }^{[14]}$ found that a common barrier to enrollment in cardiac rehab programs was lack of physician referral, even though Medicare reimburses these programs. The literature revealed that additional barriers included patient demographics, patient refusal (due to various reasons), lack of perception of need, lack of transportation and family support ${ }^{[14]}$. One study ${ }^{[13]}$ suggested the need for changes to the system to enhance referrals, to reduce barriers for prospective patients, and to consider factors that would enhance completion of the HF program that could be incorporated into the HFHEP.

In order to address the "no transportation" barrier and increase program participation, automated home monitoring equipment could be added to the current HFHEP to increase participation and effectiveness. A study by Weintraub ${ }^{[11]}$ combined automated home monitoring equipment to a nurse managed intervention that included an initial education component and weekly phone calls with a nurse manager over a 90-day period. Automated tele-monitoring equipment allowed daily measurement and transmission of blood pressure, pulse rate, and weight was added for the intervention group. At 90 days, the intervention group had a statistically significant decrease in readmissions, $p=0.05$.

The unexpected finding of prolonged LOS reflects increased costs of care related to LOS, and will receive further consideration by the health care facility where the research was conducted. 


\subsection{Limitations}

This study was performed at the completion of the first year of a disease management program in a single Mid-western community hospital and only patients with a primary diagnosis of HF were included which limited the sample size. Though patients discharged with a secondary diagnosis of HF may have utilized the intervention, they were not included in this study. Not all subjects had a value for LVEF in their medical record so some data was missing.

\subsection{Conclusions and recommendations for practice}

The HFHEP was an effective intervention to prevent 30-day readmission for patients who were discharged with a primary diagnosis of HF; however, the number of participants was low and statistical significance was not achieved. Nursing home and assisted living residents comprised $25 \%$ of the sample during the intervention year and these patients did not participate in the intervention. The referral process was a barrier to capturing all of the patients who qualified to participate in the intervention. Severity of illness, as evidenced by LVEF, did not influence participation in the intervention or readmissions. Recommendations for practice include the addition of a home tele-monitoring component to the current intervention and expanding the intervention to other health care facilities who desire to improve the quality of life of patients with heart failure and lower readmission outcomes.

It is also recommended that educational sessions be provided for nursing home and assisted living personnel regarding best practices for the management of HF, and the referral process be automated during the inpatient stay so that all potential program participants will be included. If transportation is a barrier, alternative transportation should be arranged for the participant or the intervention should be provided utilizing tele-health strategies.

Recommendations for future research include additional years of data collection from the intervention to capture a larger sample size. Nursing home residents should be excluded from future study due to their inability to participate in the intervention. Correlations between additional variables and their potential impact on readmission and participation should be explored. Specific data with regard to refusal to participate should be collected. Further investigation of LOS greater than 6 days should be conducted to determine if there are opportunities for intervention by the participating hospital.

\section{References}

[1] Bakan G, Akyo A. Theory-guided interventions for adaptation to heart failure. Journal of Advanced Nursing 2008; 61(6): 596-608. http://dx.doi.org/10.1111/j.1365-2648.2007.04489.x

[2] Zaya M, Phan A, Schwarz E. The dilemma causes and approaches to avoid recurrent hospital readmissions for patients with chronic heart failure. Heart Failure Review. 2012; 17(3): 345-353. http://dx.doi.org/10.1007/s10741-011-9256-0

[3] Velez M, Westerfeldt B, Rahko P. Why it pays for hospitals to initiate a heart failure disease management program. Disease Management and Health Outcomes. 2008; 16(3): 155-173. http://dx.doi.org/10.2165/00115677-200816030-00003

[4] Hines P, Yu K, Randall M. Preventing heart failure readmissions: is your organization prepared? Nursing Economics. 2010; 28(2): 74-86.

[5] Paul S. Hospital discharge education for patients with heart failure: what really works and what is the evidence? Critical Care Nurse. 2008; 28: 66-82.

[6] Jencks S, Williams M, Coleman E. Rehospitalizations among patients in the Medicare Fee-for-Service program. The New England Journal of Medicine. 2009; 360: 1418-1428. http://dx.doi.org/10.1056/NEJMsa0803563

[7] Janz N, Becker M. The Health Belief Model: a decade later. Health Education Quarterly. 1984; 11(1): 1-47. http://dx.doi.org/10.1177/109019818401100101

[8] Duffy J, Hoskins L, Chen M. Nonpharmacological strategies for improving heart failure outcomes in the community: a systematic review. Journal of Nursing Care Quality. 2004; 19(4): 349-360. http://dx.doi.org/10.1097/00001786-200410000-00011

[9] Krumholz H, Amatruda J, Smith G, Mattera J, Roumanis S, Radford M, et al.. Randomized trial of an education and support intervention to prevent readmission of patients with heart failure. Journal of the American College of Cardiology. 2002; 39(1): 83-89. http://dx.doi.org/10.1016/S0735-1097(01)01699-0 
[10] Dunagan C, Littenberg B, Ewald G, Jones C, Beckham Emery V, Waterman B, et al..Randomized trial of a nurse-administered, telephone-based disease management program for patients with heart failure. Journal of Cardiac Failure. 2005; 11(5): 358-365. http://dx.doi.org/10.1016/j.cardfail.2004.12.004

[11] Weintraub A, Gregory D, Patel A, Levine D, Venesy D, Perry K, et al. A multicenter randomized controlled evaluation of automated home monitoring and telephonic disease management in patients recently hospitalized for congestive heart failure: the SPAN-CHF II trial. Journal of Cardiac Failure. 2010; 16(4): 285-292. http://dx.doi.org/10.1016/j.cardfail.2009.12.012

[12] Stauffer B, Fullerton C, Fleming N, Ogola G, Herrin J, Martin Stafford P, et al. Effectiveness and cost of a transitional care program for heart failure: A prospective study with concurrent controls. Archives of Internal medicine. 2011; 171(14): 1238-1243. http://dx.doi.org/10.1001/archinternmed.2011.274

[13] Balady G, Ades P, Bittner V, Franklin B, Gordon N, Thomas R, et al. Referral, enrollment, and delivery of cardiac rehabilitation/secondary prevention programs at clinical centers and beyond: a presidential advisory from the American Heart Association. Circulation. 2011; 124: 2951-2960. http://dx.doi.org/10.1161/CIR.0b013e31823b21e2

[14] Ghisi G, Polyzotis P, Oh P, Pakosh M, Grace SL. Physician factors affecting cardiac rehabilitation referral and patient enrollment: A systematic review. Clinical Cardiology. 2013; 36(6): 323-335. http://dx.doi.org/10.1002/clc.22126 